\title{
miRNA expression profiles in Smad4-positive and Smad4-negative SW620 human colon cancer cells detected by next-generation small RNA sequencing
}

This article was published in the following Dove Press journal:

Cancer Management and Research

Wei Yan

Zhongcai Liu

Wenchao Yang

Guoyang Wu

Department of General Surgery, Zhongshan Hospital, Xiamen

University, Xiamen, Fujian 361004

People's Republic of China
Correspondence: Guoyang Wu

Department of General Surgery,

Zhongshan Hospital, Xiamen University,

20I-209 Hubin South Road, Xiamen,

Fujian 361004, People's Republic of China

Emailw13400677I16@aliyun.com
Background and aims: SMAD4, as a tumor suppressive gene in human colon cancer, inhibits the metastasis of colon adenocarcinoma cells. However, the molecular mechanisms are unclear miRNAs play an important role in the pathogenesis and progression of cancer.

Methods: In this study, a deep sequencing technique was used to screen Smad4-regulated miRNAs in human colon cancer SW620 cell line. Using a next-generation small RNA sequencing approach, we compared the miRNA expression profiles of SW620 colon cancer cells transfected with smad4 lentiviral vector with those transfected with control vector. Six samples were selected and sequenced randomly each from control group (smad4-negative cell) and Smad4 group (Smad4-positive cells). Quantitative reverse transcription-PCR (qRT-PCR) and Western blot (WB) was used to validate the results of sequencing.

Results: Smad4 reexpression significantly upregulated 43 known miRNAs and downregulated 10 known miRNAs expression. Gene Ontology and Kyoto Encyclopedia of Genes and Genomes pathway analysis of predicted miRNAs targets showed that these genes were mainly involved in protein-binding transcription factor activity, vascular smooth muscle contraction, pathways in cancer metastasis, and phosphatidylinositol 3-kinase-Akt signal pathway. qRT-PCR and WB validated the partial results of sequencing. Reexpression of Smad4 inhibited colon cancer cell migration and invasion. Smad4 reexpression increased the expression of E-cadherin (E-cad) and decreased the Vimentin (Vim) and Matrix Metalloproteinase-9 expression. Restoration of SMAD4 results in a marked decrease of Vim by inhibiting p-AKT and p-EPHA2, but significantly increased the E-cad by AKT-EPHA2 pathways.

Conclusion: Smad4 inhibits the migration and invasion ability of colon cancer cells in vitro and this is the first report of Smad4-mediated miRNA expression profiling in Smad4-positive and Smad4-negative SW620 human colon cancer cells, which may help us better understand the role of Smad4 in inhibiting the metastasis of colon cancer cells and its possible molecular mechanisms.

Keywords: Smad4, miRNA, colon cancer, next-generation sequencing

\section{Introduction}

Colon cancer is one of the most common types of tumors in humans. ${ }^{1}$ Many researchers have shown that SMAD4 is frequently lost in colon cancers, which is associated with the development of metastases and a poor prognosis. ${ }^{1,2}$ Smad 4 mutations were observed in $95 \%$ invasive and metastatic colon cancers. ${ }^{2}$ Many studies have demonstrated the Smad4 pathway has been shown to mediate the antimetastatic role in colon cancer 
metastasis. ${ }^{1-3,9-15}$ This study and other researcher's previous studies found that Smad4 was associated with poor prognosis in patients with colon cancer and inhibited tumor metastasis through multiple processes, including the inhibition of apoptosis and inhibition of the epithelial-mesenchymal transition (EMT) process, and phosphatidylinositol 3-kinase-Akt (PI3K-Akt) signaling pathway. ${ }^{1-3,9,15,25}$ However, the exact mechanism remains unclear. Therefore, studying the role of Smad4 in tumor metastasis is essential for developing more effective treatments and improving colon cancer patients' prognosis.

Recently, many miRNAs-related metastasis gene have been identified. ${ }^{4,5}$ miRNAs are 20-22 nucleotide endogenous noncoding RNAs, which repress target gene expression at the post-transcriptional level and are now considered to be major regulators of gene expression for at least $30 \%$ of human genes. $^{5-7}$ Functional studies indicate that miRNAs have important functions in tumor progression, especially recurrence, drug resistance, and metastasis. ${ }^{4-8} \mathrm{~A}$ lot of researchers have provided strong evidence that miRNAs play an important role during tumorigenesis and tumor progression. ${ }^{4-8}$

Given that miRNAs play a significant role in gene regulation and tumorigenesis, and Smad4 is the central component of the TGF- $\beta$ and bone morphogenetic protein signaling pathway which plays an important role in cancer progression, miRNAs might interact with the Smad superfamily in tumors. ${ }^{6,7}$ Thus, we hypothesized that Smad4 may mediate the activation or inhibition of miRNAs, which could influence colon cancer metastasis. Until now, there are no reports about the miRNA expression profile following smad4 reexpression in a colon cancer cell model. In this study, we profiled the miRNAs of the SW620 colon cancer cell line transfected with smad4 lentiviral vector, which can get stable reexpression of Smad4. Fifty-three known miRNAs were found to be differentially expressed. Some of the differentially expressed miRNAs might be potential biomarkers for colon cancer. Thus, we provide a new context to study the mechanism by which Smad4 inhibited tumor metastasis.

\section{Materials and methods}

\section{Establishment of colon cancer cell lines with stable overexpression of Smad4}

Human colon adenocarcinoma cell line SW620, which was derived from lymph node metastasis and does not express Smad4, was obtained from American Type Culture Collection (Manassas, VA) and was cultured in RPMI 1640 media supplemented with $10 \%$ FBS. The full-length coding region of Smad4 gene was amplified with PCR, and the amplified fragment was cloned into the lentiviral vector GV248. The recombinant lentiviral vector was transfected into $293 \mathrm{~T}$ cells for packaging the virus, which was then transfected into colon cancer SW620 cells. The stably transfected cells were selected with G418, and the cellular expressions of Smad4 mRNA and protein were detected using quantitative PCR and Western blotting (WB).

\section{smRNA library construction and next- generation sequencing}

Six samples were selected randomly each from control group (smad4-negative cells) and Smad4 group (Smad4-positive cells), and sequenced by second-generation illumina highthroughput sequencing, screened for differentially expressed miRNA. Briefly, total RNA was collected from three pairs of Smad4-positive cells and matched smad4-negative cells by using TRIzol reagent (Life Technologies, Carlsbad, CA, USA). Qualified RNA with A260/A280 ratio more than 1.8 was subsequently assessed for RNA integrity by conducting the DNA chip assay. smRNA libraries were constructed by using a smRNA sample prep kit (Illumina, San Diego, CA, USA) following the standard protocols. Eventually, the libraries which were constructed from three independent Smad4positive cells and three matched independent smad4-negative cells were sequenced by applying Illumina HiSeq 2500 (Illumina, USA) at GENE DENOVO (Guangzhou, People's Republic of China) strictly following the standard protocols.

\section{Data analysis}

Consensus Assessment of Sequence and Variation (version 1.8.2; all Illumina, Inc.), Illumina HiSeq Control software (version 2.0.12), and Real-Time Analysis software (version 1.12.4.2) were applied to analyze the raw data. Differential expression levels of miRNA were calculated and standardized to reads per million, as follows: Counts/total count of each sample $\times 1$ million. Target genes of differentially expressed miRNAs were predicted using the TargetScan database, miRDB database, and miRanda database, which were downloaded from http://www.targetscan.org/, http://www. mirdb.org/, and http://www.microrna.org/, respectively. The predicted genes underwent Gene Ontology (GO; http://www. geneontology.org/) and Kyoto Encyclopedia of Genes and Genomes (KEGG; http://www.genome.jp/kegg/) pathway analyses using the functional annotation tools of the Database from Annotation, Visualization, and Integrated Discovery (DAVID; http://david.abcc.Ncifcrf.gov/). The enrichment of GO terms and KEGG pathways were selected using a cutoff standard of $P<0.01$ and false discovery rate $<1$. 


\section{Quantitative reverse transcription- polymerase chain reaction ( $\mathrm{qRT}-\mathrm{PCR}$ )}

Smad4-negative cells and Smad4-positive cells were collected. Following total RNA isolation, cDNA were synthesized using a miDETECT A Track ${ }^{\mathrm{TM}}$ miRNA qRT-PCR Starter kit (RIOB IO Biotechnology Co., Ltd., Guangzhou, People's Republic of China). All the miRNA primers (miDETECT A TrackTM miRNA qPCR Primer Set, including hsa-miR323b-3p, hsa-miR-323b-3p, hsa-miR-371a-5p, hsa-miR708-3p, hsa-miR-6852-5p, hsa-miR-767-5p, hsa-miR-382-5p, hsa-miR-514a-3p, hsa-miR-1289, hsa-miR-1537-5p, hsamiR-23c, hsa-miR-3199, hsa-miR-3619-5p, hsa-miR-3657, hsa-miR-548u, hsa-miR-5699-5p, hsa-miR-874-5p) and control primer (miDETECT A TrackTM U6 qPCR Primer Set) were also purchased from this company. qPCR was performed on an ABI 7500 real-time PCR system (Applied Biosystems, Carlsbad, CA, USA) according to the manufacturer's protocol by miDETECT A TrackTM miRNA qPCR kit (RIOB IO Biotechnology Co., Ltd.). Dissociation curve analysis was used to verify the generation of single products. U6 was used as a reference gene, and all reactions were repeated three times. Data were analyzed using the ABI 7500 Prism sequence detection software (Applied Biosystems) and the relative expression was calculated using the $2{ }^{\Delta \Delta C q}$ method.

\section{WB}

Total cell extracts were prepared in a lysis buffer (Solarbio, Peking, People's Republic of China). After 10 minutes on ice, cell debris was removed by centrifugation at 10,000 $\mathrm{g}$ for 10 minutes. Protein concentrations were determined by the BCA method (Sigma, Santa Clara, CA, USA) using BSA as a control. Proteins were separated by SDS-PAGE and then transferred onto polyvinylidene difluoride membranes (Minipore, Billerica, MA, USA), which were treated with the appropriate primary antibodies (Abcam, UK). Antibody-bound proteins were detected by a chemiluminescence method according to the manufacturer's protocol (CB Company, Murray, UT, USA).

\section{Migration and invasion assays}

Human colon cancer cell line SW480, which does not express Smad4, and RKO, HCT116, LOVO, and HCT8 cell lines were obtained from American Type Culture Collection and were cultured in RPMI 1640 media supplemented with 10\% FBS. Transwell Cell Migration and Invasion Assay kit (BD Biosciences, Franklin Lakes, NJ, USA) was used to analyze cell migration and invasion ability strictly following the standard protocols. Generally, a transwell chamber was placed into a 24-well plate and was coated with $30 \mu \mathrm{L}$ matrigel and was incubated for 40 minutes at $37^{\circ} \mathrm{C}$ for the invasion assay. In both transwell assay, stably transfected cells were trypsinized and seeded in chambers and cultured, while $600 \mu \mathrm{L}$ medium was added to the lower chamber. About 24 hours later, migrated cells were fixed with $100 \%$ methanol for 30 minutes. Nonmigrated cells were removed. Then cells on bottom surface of the membrane were stained by crystal violet for 20 minutes. Cell images were obtained under a phase-contrast microscope (Olympus, Tokyo, Japan).

\section{Statistical analysis}

Statistical analysis was performed using SPSS statistical software (version 17.0; SPSS, Inc., Chicago, IL, USA). The significant difference between the two groups was analyzed by one-way analysis of variance using paired $t$-test. Data are expressed as mean $\pm \mathrm{SD}$. $P<0.05$ was considered as significant difference.

\section{Results}

\section{Stable overexpression of Smad4 in SW620 colorectal cancer cell line}

First, we chose the SW620 human colon cancer cell model, which is null in Smad4 expression. To get the stable expression of Smad4 gene, we transfected the lentiviral Smad4 vector into the cells and got the stable cell model, SW620 Smad4, which reexpressed the gene of Smad4. As shown in Figure 1A, B, we performed WB and qRT-PCR and found that transfection of Samd4 activated the reexpression of Smad4.

\section{miRNA expression profiles following stable Smad4 re-expression}

To identify the difference in miRNA expression in control group (smad4-negative cells) and Smad4 group (Smad4positive cells), we used the second-generation illumina highthroughput sequencing technology and screened differentially expressed miRNAs. We found that 53 differentially expressed miRNAs (including 43 upregulated and 10 downregulated known miRNAs, respectively) in the SW620-Smad4 cells in contrast to the SW620-control cells. Table 1 summarizes the 53 miRNAs that show the significant differences. We also found the novel differentially miRNAs expression profiles of the two cell lines, which is shown in Table 2, especially, novel-m0099-5p, novel-m0100-5p, novel-m0101-5p, novel$\mathrm{m} 0102-5 \mathrm{p}$, and novel-m0103-5p were reduced by about 63 times and novel-m0188-5p, novel-m0030-5p, novel$\mathrm{m} 0227-3 \mathrm{p}$, novel-m0228-3p, and novel-m0251-5p were increased by about $8,16,23,23,10$ times, respectively. 
A

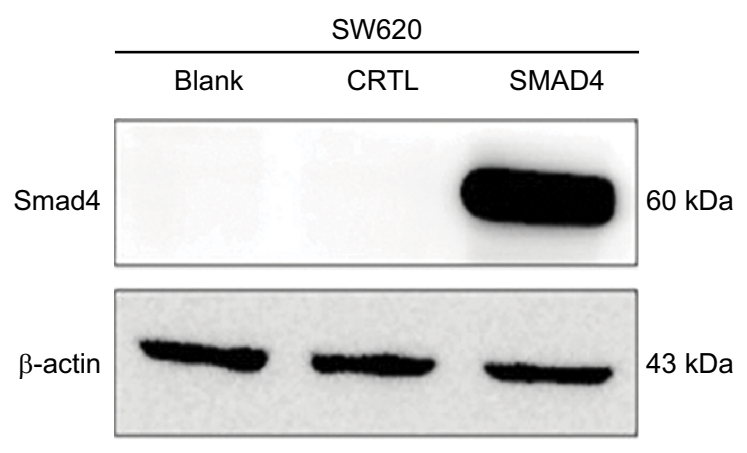

B

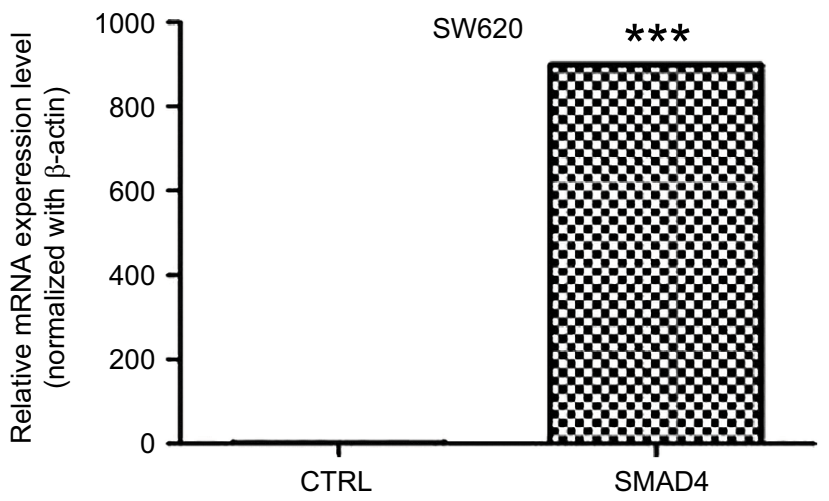

Figure I Validation of Smad4 reexpression in Smad4-negative SW620 cells by WB and qRT-PCR.

Notes: (A) Expression of Smad4 by WB. Blank: null SW620 cells. CTRL: empty vector-infected SW620 cells. SMAD4: Smad4 vector-infected SW620 cells. $\beta$-Actin was used as an internal control. (B) qRT-PCR detection of Smad 4 mRNA expression level in SW620 cells following Smad4 reexpression. $* * * P<0.001$.

Abbreviations: $q R T-P C R$, quantitative reverse transcription-polymerase chain reaction; WB, Western blot.

\section{Validation of differentially expressed miRNAs}

miRNA-specific qRT-PCR was performed to validate the differentially expressed miRNAs. As shown in Figure 2A, the expression levels of hsa-miR-323b-3p, hsa-miR-323b-3p, hsa-miR-371a-5p, hsa-miR-708-3p, hsa-miR-6852-5p, hsamiR-767-5p, hsa-miR-382-5p, and hsa-miR-514a-3p were significantly upregulated in SW620-Smad4 cells, whereas, as shown in Figure 2B, hsa-miR-1289, hsa-miR-1537-5p, hsamiR-23c, hsa-miR-3199, hsa-miR-3619-5p, hsa-miR-3657, hsa-miR-548u, hsa-miR-5699-5p, and hsa-miR-874-5p were downregulated. These findings are consistent with the results of the miRNAs sequencing results.

\section{GO analysis of predicted target genes of differentially expressed miRNAs}

To investigate the potential predicted target gene enrichment in cellular component (Table 3), molecular function (Table 4),
Table I Differentially expressed miRNAs between stable Smad4 overexpression cells and controls $(P<0.05$, fold change $>2)$

\begin{tabular}{|c|c|c|c|}
\hline miRNA & $\begin{array}{l}\text { Fold } \\
\text { change }\end{array}$ & $\begin{array}{l}\text { Type of } \\
\text { regulation }\end{array}$ & $P$-value \\
\hline hsa-miR-I-3p & 3.0300481 & $U_{p}$ & $1.68 \mathrm{E}-05$ \\
\hline hsa-miR-I05-5p & 9.6320385 I & $U_{p}$ & 0.004776827 \\
\hline hsa-miR-I250-5p & 2.76590929 & $U_{p}$ & 0.016764887 \\
\hline hsa-miR-127-3p & 3.51000858 & $U_{p}$ & 0.017063522 \\
\hline hsa-miR-129-I-3p & 20.2566667 & $U_{p}$ & $6.26 \mathrm{E}-07$ \\
\hline hsa-miR-133a-3p & 2.82510445 & $U_{p}$ & 0.000749984 \\
\hline hsa-miR-133a-5p & 2.39407718 & Up & 0.0413216 \\
\hline hsa-miR-136-3p & 2.87518716 & $U_{p}$ & 0.04961782 \\
\hline hsa-miR-139-5p & 2.75808538 & $U_{p}$ & 0.039079472 \\
\hline hsa-miR-203a-3p & 2.62724607 & Up & $8.05 \mathrm{E}-05$ \\
\hline hsa-miR-203b-3p & 2.68843604 & Up & 0.000114467 \\
\hline hsa-miR-3|49 & 2.2304999 & Up & 0.02335124 \\
\hline hsa-miR-323b-3p & 58.3133333 & $U_{p}$ & $8.64 \mathrm{E}-05$ \\
\hline hsa-miR-338-5p & 2.78646827 & $U_{p}$ & 0.01011844 \\
\hline hsa-miR-3656 & 3.98101266 & Up & 0.013411597 \\
\hline hsa-miR-365b-5p & 23.7966667 & $U_{p}$ & $4.75 \mathrm{E}-05$ \\
\hline hsa-miR-369-3p & 4.58094845 & Up & 0.015997829 \\
\hline hsa-miR-37la-5p & 41.9733333 & Up & 0.003763824 \\
\hline hsa-miR-376c-3p & 2.58784235 & Up & 0.047594468 \\
\hline hsa-miR-379-5p & 3.24035036 & $U_{p}$ & 0.014697283 \\
\hline hsa-miR-38I-3p & 2.17839525 & $U_{p}$ & 0.040985364 \\
\hline hsa-miR-382-5p & 17.7724868 & $U_{p}$ & 0.013815686 \\
\hline hsa-miR-3909 & 2.02610176 & Up & 0.003951009 \\
\hline hsa-miR-409-3p & 4.93321119 & $U_{p}$ & 0.009351995 \\
\hline hsa-miR-43I-5p & 3.59492282 & $U_{p}$ & 0.021180118 \\
\hline hsa-miR-449a & 2.88279908 & $U_{p}$ & 0.01816137 \\
\hline hsa-miR-455-3p & 2.13778874 & $U_{p}$ & 0.043774928 \\
\hline hsa-miR-4799-5p & 20.2566667 & $U_{p}$ & $6.26 \mathrm{E}-07$ \\
\hline hsa-miR-483-3p & 4.37163969 & Up & 0.026865568 \\
\hline hsa-miR-483-5p & 4.70802709 & $U_{p}$ & 0.010097822 \\
\hline hsa-miR-487b-3p & 10.1258278 & $U_{p}$ & 0.035457763 \\
\hline hsa-miR-509-3p & 9.46230159 & Up & 0.040214237 \\
\hline hsa-miR-5|4a-3p & 15.8852097 & $U_{p}$ & 0.02453244 \\
\hline hsa-miR-548am-3p & $2.4936 \mid 435$ & Up & 0.043489873 \\
\hline hsa-miR-550a-5p & 2.0507075 I & $U_{p}$ & 0.021124708 \\
\hline hsa-miR-656-3p & 46.5566667 & Up & $1.21 \mathrm{E}-06$ \\
\hline hsa-miR-6852-5p & 20.2566667 & $U_{p}$ & $6.26 \mathrm{E}-07$ \\
\hline hsa-miR-708-3p & 38.0533333 & $U_{p}$ & 0.000659703 \\
\hline hsa-miR-744-5p & 29.8433333 & $U_{p}$ & $2.92 \mathrm{E}-05$ \\
\hline hsa-miR-758-5p & 20.2566667 & $U_{p}$ & $6.26 \mathrm{E}-07$ \\
\hline hsa-miR-875-5p & 46.6033333 & $U_{p}$ & 0.000407778 \\
\hline hsa-miR-889-3p & 2.97430749 & $U_{p}$ & 0.012912535 \\
\hline hsa-miR-767-5p & 22.6434519 & $U_{p}$ & 0.021766387 \\
\hline hsa-miR-I289 & 2.10708403 & Down & $0.0480 \mid 4258$ \\
\hline hsa-miR-I537-5p & 12.0816166 & Down & 0.032522333 \\
\hline hsa-miR-23c & 17.019999 & Down & 0.000233603 \\
\hline hsa-miR-3199 & 17.019999 & Down & 0.000233603 \\
\hline hsa-miR-3619-5p & 8.12123642 & Down & $0.044 \mid 23068$ \\
\hline hsa-miR-3657 & 2.25696259 & Down & $0.029294 \mid 54$ \\
\hline hsa-miR-548u & 2.16192045 & Down & 0.035163924 \\
\hline hsa-miR-5699-5p & 2.59881075 & Down & 0.010750283 \\
\hline hsa-miR-874-5p & $3.764 \mid 1255$ & Down & 0.007516669 \\
\hline hsa-miR-4684-3p & 2.80937387 & Down & 0.03699219 \\
\hline
\end{tabular}


and biological process (Table 5), GO analysis was performed with the differentially expressed miRNAs following Smad4 reexpression. The results suggested that a lot of functional signaling pathways were enriched, including those involved in the regulation of cellular metabolic process, anatomical structure development, developmental process, and regulation of

Table 2 The list of differentially expressed novel miRNAs between stable Smad4 overexpression cells and controls $(P<0.05$, fold change >2)

\begin{tabular}{|l|l|l|l|}
\hline miRNA & $\begin{array}{l}\text { Fold } \\
\text { change }\end{array}$ & $\begin{array}{l}\text { Type of } \\
\text { regulation }\end{array}$ & P-value \\
\hline novel-m0012-5p & 11.537432 & Down & 0.018215425 \\
novel-m0080-5p & 29.89626 & Down & $1.43 \mathrm{E}-05$ \\
novel-m0084-5p & 3.0550489 & Down & 0.022574517 \\
novel-m0099-5p & 63.828429 & Down & 0.00013912 \\
novel-m0100-5p & 63.828429 & Down & 0.00013912 \\
novel-m010I-5p & 63.828429 & Down & 0.00013912 \\
novel-m0102-5p & 63.828429 & Down & 0.00013912 \\
novel-m0103-5p & 63.828429 & Down & 0.00013912 \\
novel-m0I79-5p & $2.889405 I$ & Down & 0.027729009 \\
novel-m0333-3p & 4.85719837 & Down & 0.011391107 \\
novel-m0336-3p & 4.85719837 & Down & 0.011391107 \\
novel-m0246-3p & 2.13677497 & Down & 0.020091995 \\
novel-m0190-5p & 2.88940513 & Down & 0.027729009 \\
novel-m0188-5p & 8.014228 & Up & 0.036847661 \\
novel-m0030-5p & 16.71333 & Up & 0.000194285 \\
novel-m0227-3p & 23.84 & Up & 0.001772445 \\
novel-m0228-3p & 23.84 & Up & 0.001772445 \\
novel-m025I-5p & 10.19173 & Up & 0.025869475 \\
\hline
\end{tabular}

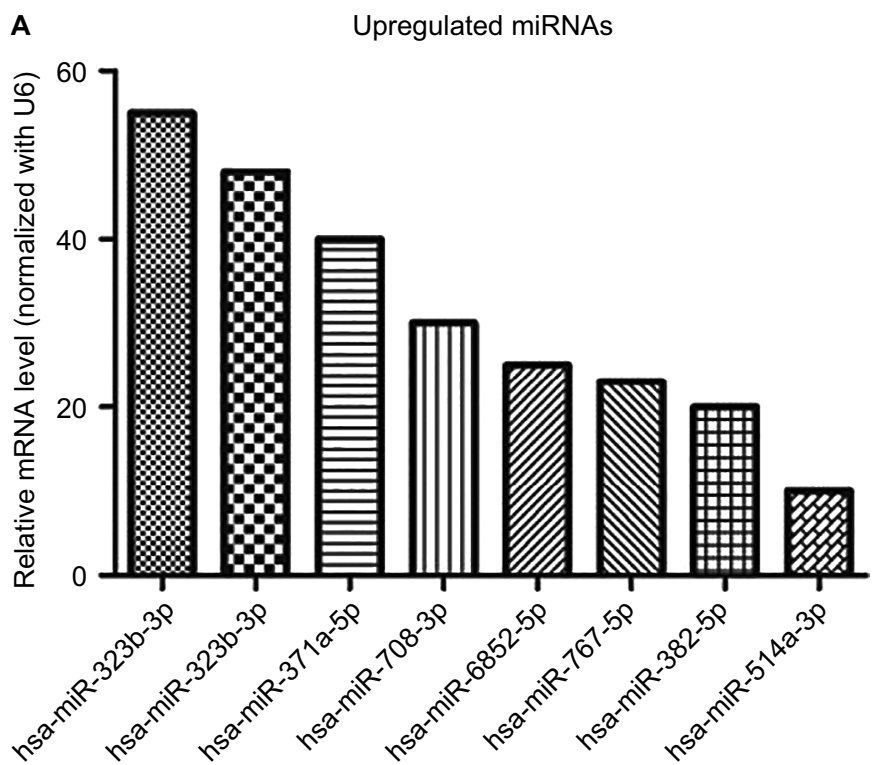

molecular-binding processes. In addition, the intracellular part exhibited the most increased enrichment of GO terms in Smad4 reexpression cell (Table 3).

\section{KEGG pathway analysis of predicted target genes of differentially expressed miRNAs}

KEGG pathway analysis was also performed with the differentially expressed miRNAs following Smad4 reexpression, which indicated that vascular smooth muscle contraction pathways and pathways in cancer metastasis were changed and corresponded to differentially expressed transcripts (Table 6).

\section{Validation of partial sequencing data analysis results}

To demonstrate our hypothesis, we first screened the expression of Smad4 in six kinds of colon cancer cells including SW480, SW620, RKO, HCT116, LOVO, and HCT8. As shown in Figure 3A, the expression level of Smad4 was null in SW480 and SW620 cells, whereas RKO, HCT116, LOVO, and HCT8 cells had higher expression levels of Smad4. Then, we also transfected the lentiviral Smad4 vector into the SW480 cells and got the stable cell model, SW480 Smad4 (Figure 3B). Smad4 reexpression inhibited cell migration and invasion in SW480 (Figure 4A, B)

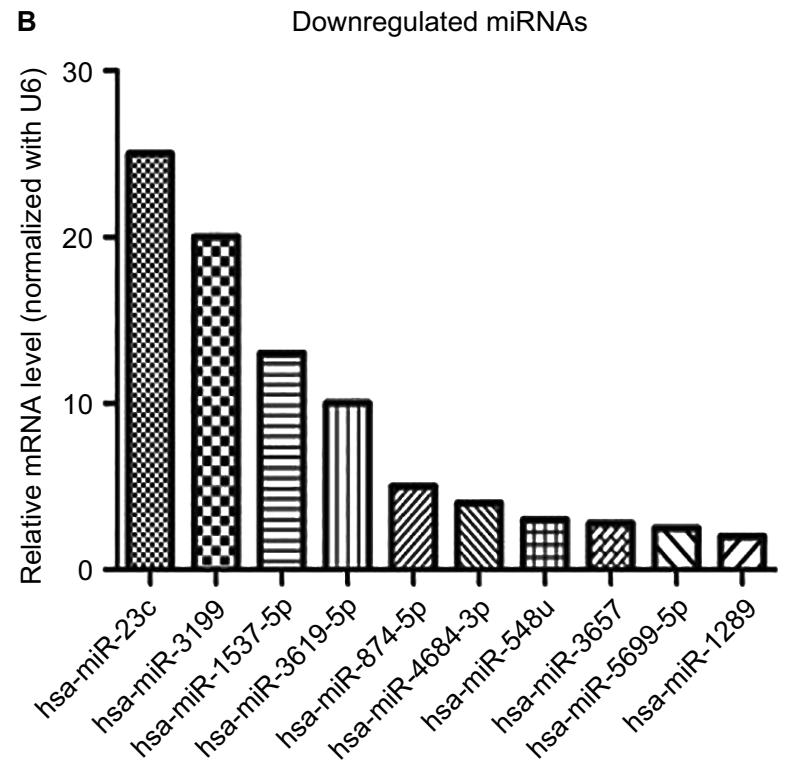

Figure 2 Validation of some miRNA sequencing results by qRT-PCR.

Notes: (A) Eight upregulated miRNAs in the stable Smad4 overexpression cells group; (B) Ten downregulated miRNAs in the stable Smad4 overexpression cells group. U6 was used as a normalization control. Data represent the mean \pm SD. of three biological replicates. ${ }^{*} P<0.05$.

Abbreviation: $q R T-P C R$, quantitative reverse transcription-polymerase chain reaction. 
Table 3 The most significantly enriched GO terms for cellular component for the predicted targets of the differentially expressed miRNAs following Smad4 reexpression

\begin{tabular}{|c|c|c|c|c|}
\hline GO term & $\begin{array}{l}\text { Cluster frequency } \\
\text { (out of } 31,172 \text { genes) }\end{array}$ & $\begin{array}{l}\text { Genome frequency of } \\
\text { use (out of } 50,073 \text { genes) }\end{array}$ & $P$-value & $\begin{array}{l}\text { Corrected } \\
\text { P-value }\end{array}$ \\
\hline intracellular part & $20,076(64.4 \%)$ & $29,5 \mid 4(58.9 \%)$ & $1.17 \mathrm{e}-222$ & $7.5 \mid \mathrm{le}-22 \mathrm{I}$ \\
\hline organelle & 19,075 (61.2\%) & $27,978(55.9 \%)$ & $6.33 e-208$ & $4.05 e-206$ \\
\hline cytoplasm & $14,727(47.2 \%)$ & $21,188(42.3 \%)$ & $6.13 e-183$ & $3.92 \mathrm{e}-18 \mathrm{I}$ \\
\hline intracellular organelle & $16,265(52.2 \%)$ & $23,970(47.9 \%)$ & $2.35 \mathrm{e}-136$ & $1.50 \mathrm{e}-134$ \\
\hline cytoplasmic part & $9,278(29.8 \%)$ & $13,032(26.0 \%)$ & $5.87 \mathrm{e}-136$ & $3.76 \mathrm{e}-134$ \\
\hline membrane-bounded organelle & $14,858(47.7 \%)$ & $21,792(43.5 \%)$ & $1.26 \mathrm{e}-128$ & $8.07 \mathrm{e}-127$ \\
\hline intracellular membrane-bounded organelle & $14,858(47.7 \%)$ & $21,792(43.5 \%)$ & $1.26 \mathrm{e}-128$ & $8.07 e-127$ \\
\hline intracellular & $20,779(66.7 \%)$ & $31,419,62.7 \%)$ & $4.35 \mathrm{e}-119$ & $2.78 \mathrm{e}-117$ \\
\hline cell part & $23,565(75.6 \%)$ & $36,179(72.3 \%)$ & $3.80 \mathrm{e}-101$ & $2.43 e-99$ \\
\hline cell & $23,566((75.6 \%)$ & $36,183(72.3 \%)$ & $6.99 \mathrm{e}-101$ & $4.47 e-99$ \\
\hline cytosol & $4,639(14.9 \%)$ & 6,275 (12.5\%) & $6.12 \mathrm{e}-97$ & $3.91 \mathrm{e}-95$ \\
\hline endomembrane system & 3,602 (II.6\%) & $4,94 \mid(9.9 \%)$ & $3.82 \mathrm{e}-62$ & $2.44 \mathrm{e}-60$ \\
\hline nucleus & $\mathrm{II}, 000(35.3 \%)$ & $16,350(32.7 \%)$ & $1.58 \mathrm{e}-59$ & $1.01 \mathrm{e}-57$ \\
\hline cytoskeleton & $2,394(7.7 \%)$ & $3,296(6.6 \%)$ & $8.56 \mathrm{e}-39$ & $5.47 e-37$ \\
\hline organelle part & 3,724 (II.9\%) & $5,298(10.6 \%)$ & $1.36 \mathrm{e}-38$ & $8.75 e-37$ \\
\hline intracellular organelle part & 3,724 (1I.9\%) & $5,298(10.6 \%)$ & $1.36 \mathrm{e}-38$ & $8.75 e-37$ \\
\hline
\end{tabular}

Note: Terms from the Ontology with $P$-value as good or better than I.

Abbreviation: GO, Gene Ontology.

Table 4 The most significantly enriched GO terms for molecular function for the predicted targets of the differentially expressed miRNAs following Smad4 reexpression

\begin{tabular}{|c|c|c|c|c|}
\hline GO term & $\begin{array}{l}\text { Cluster frequency } \\
\text { (out of } 33,646 \text { genes) }\end{array}$ & $\begin{array}{l}\text { Genome frequency of } \\
\text { use (out of } 58,624 \text { genes) }\end{array}$ & $P$-value & $\begin{array}{l}\text { Corrected } \\
\text { P-value }\end{array}$ \\
\hline binding & $18,59 \mid(55.3 \%)$ & $29,447(50.2 \%)$ & $4.79 \mathrm{e}-176$ & $3.02 \mathrm{e}-174$ \\
\hline protein binding & $4,776(14.2 \%)$ & $6,698(11.4 \%)$ & $2.12 \mathrm{e}-137$ & $1.34 \mathrm{e}-135$ \\
\hline enzyme binding & $2,345(7.0 \%)$ & $3,122(5.3 \%)$ & $9.80 \mathrm{e}-100$ & $6.17 \mathrm{e}-98$ \\
\hline nucleic acid binding & 6,696 (19.9\%) & $10,240(17.5 \%)$ & $5.83 e-74$ & $3.67 \mathrm{e}-72$ \\
\hline organic cyclic compound binding & $6,696(19.9 \%)$ & $10,240(17.5 \%)$ & $5.83 e-74$ & $3.67 \mathrm{e}-72$ \\
\hline heterocyclic compound binding & 6,696 (19.9\%) & $10,240(17.5 \%)$ & $5.83 e-74$ & $3.67 e-72$ \\
\hline ion binding & $12,255(36.4 \%)$ & $19,725(33.6 \%)$ & $7.43 \mathrm{e}-62$ & $4.68 \mathrm{e}-60$ \\
\hline DNA binding & $4,403(13.1 \%)$ & 6,729 (1I.5\%) & $1.19 \mathrm{e}-46$ & $7.55 \mathrm{e}-45$ \\
\hline protein binding transcription factor activity & $1,023(3.0 \%)$ & $\mathrm{I}, 373(2.3 \%)$ & $8.46 e-4 I$ & $5.33 e-39$ \\
\hline transcription factor binding & $817(2.4 \%)$ & $\mathrm{I}, 104(\mathrm{I} .9 \%)$ & $3.82 \mathrm{e}-3 \mathrm{I}$ & $2.40 \mathrm{e}-29$ \\
\hline lipid binding & $\mathrm{I}, 096(3.3 \%)$ & $\mathrm{I}, 533(2.6 \%)$ & $4.59 \mathrm{e}-31$ & $2.89 \mathrm{e}-29$ \\
\hline RNA binding & $2,705(8.0 \%)$ & $4,107(7.0 \%)$ & $7.84 \mathrm{e}-3 \mathrm{I}$ & $4.94 \mathrm{e}-29$ \\
\hline cytoskeletal protein binding & $\mathrm{I}, 6 \mathrm{II}(4.8 \%)$ & $2,365(4.0 \%)$ & $5.58 \mathrm{e}-28$ & $3.5 \mathrm{Ie}-26$ \\
\hline enzyme regulator activity & $\mathrm{I}, 688(5.0 \%)$ & $2,5 \mid 8(4.3 \%)$ & $2.50 \mathrm{e}-24$ & $1.58 \mathrm{e}-22$ \\
\hline molecular function regulator & $\mathrm{I}, 688(5.0 \%)$ & $2,518(4.3 \%)$ & $2.50 \mathrm{e}-24$ & $1.58 \mathrm{e}-22$ \\
\hline nucleic acid binding transcription factor activity & $2,103(6.3 \%)$ & $3,197(5.5 \%)$ & $1.29 \mathrm{e}-23$ & $8.15 \mathrm{e}-22$ \\
\hline transferase activity & $3,64 \mathrm{I}(10.8 \%)$ & $5,885(10.0 \%)$ & $1.05 \mathrm{e}-13$ & $6.67 \mathrm{e}-12$ \\
\hline histone binding & $256(0.8 \%)$ & $344(0.6 \%)$ & $3.29 \mathrm{e}-11$ & $2.07 \mathrm{e}-09$ \\
\hline phosphatase activity & $578(1.7 \%)$ & $853(1.5 \%)$ & $2.49 \mathrm{e}-10$ & $1.56 \mathrm{e}-08$ \\
\hline
\end{tabular}

Note: Terms from the function ontology with $P$-value as good or better than I.

Abbreviation: GO, Gene Ontology.

and SW620 (Figure 5A, B) cells, which is independent of TGF- $\beta 1$. Smad4 reexpression increased the expression of E-cadherin (E-cad), and decreased the Vimentin (Vim) and Matrix Metalloproteinase-9 (MMP-9) expression (Figures 4C and 5C). Restoration of SMAD4 results in a marked decrease of Vim by inhibiting p-AKT and p-EPHA2, but significantly increased the E-cad by AKT-EPHA2 pathways in colon cancer SW480 cells (Figure 6A). We got the same phenomenon in colon cancer SW620 cells following stable Smad4 reexpression (Figure 6B). 
Table 5 The most significantly enriched GO terms for molecular biological process for the predicted targets of the differentially expressed miRNAs following Smad4 reexpression

\begin{tabular}{|c|c|c|c|c|}
\hline GO term & $\begin{array}{l}\text { Cluster frequency } \\
\text { (out of } 30,854 \text { genes) }\end{array}$ & $\begin{array}{l}\text { Genome frequency of } \\
\text { use (out of } 51,743 \text { genes) }\end{array}$ & $P$-value & $\begin{array}{l}\text { Corrected } \\
\text { P-value }\end{array}$ \\
\hline cellular process & 21,945 (7I.1\%) & $34,615(66.9 \%)$ & $2.03 e-135$ & $3.46 \mathrm{e}-133$ \\
\hline single-organism process & I6,897 (54.8\%) & $26,171(50.6 \%)$ & $6.57 \mathrm{e}-119$ & $1.11 \mathrm{e}-116$ \\
\hline developmental process & $4,416(14.3 \%)$ & $6,062(11.7 \%)$ & $2.07 e-115$ & $3.52 \mathrm{e}-113$ \\
\hline single-organism cellular process & $13,739(44.5 \%)$ & $21,184(40.9 \%)$ & $2.93 \mathrm{e}-91$ & $4.98 \mathrm{e}-89$ \\
\hline metabolic process & $15,628(50.7 \%)$ & $24,384(47.1 \%)$ & $2.19 \mathrm{e}-85$ & $3.72 \mathrm{e}-83$ \\
\hline biosynthetic process & $7,285(23.6 \%)$ & $10,77 \mid(20.8 \%)$ & $1.50 \mathrm{e}-82$ & $2.56 \mathrm{e}-80$ \\
\hline cellular metabolic process & $13,537(43.9 \%)$ & $20,960(40.5 \%)$ & $7.92 \mathrm{e}-8 \mathrm{I}$ & $1.34 \mathrm{e}-78$ \\
\hline anatomical structure development & $3,194(10.4 \%)$ & $4,389(8.5 \%)$ & $1.82 \mathrm{e}-80$ & 3.10e-78 \\
\hline cellular component organization & $5,065(16.4 \%)$ & 7,347 (14.2\%) & $4.72 \mathrm{e}-7 \mathrm{I}$ & $8.02 \mathrm{e}-69$ \\
\hline cellular component organization or biogenesis & $5,107(16.6 \%)$ & $7,419(14.3 \%)$ & $2.80 \mathrm{e}-70$ & $4.76 \mathrm{e}-68$ \\
\hline cellular nitrogen compound metabolic process & $8,866(28.7 \%)$ & $13,540(26.2 \%)$ & $1.38 \mathrm{e}-59$ & $2.35 \mathrm{e}-57$ \\
\hline nitrogen compound metabolic process & 9,067 (29.4\%) & $|3,87|(26.8 \%)$ & $3.37 e-59$ & $5.74 \mathrm{e}-57$ \\
\hline cell differentiation & $1,839(6.0 \%)$ & $2,476(4.8 \%)$ & $2.66 \mathrm{e}-55$ & $4.53 \mathrm{e}-53$ \\
\hline single-organism developmental process & $2,283(7.4 \%)$ & $3,161(6.1 \%)$ & $1.61 \mathrm{e}-52$ & $2.75 e-50$ \\
\hline Localization & $7,104(23.0 \%)$ & 10,785 )20.8\%) & $8.54 \mathrm{e}-5 \mathrm{I}$ & $1.45 \mathrm{e}-48$ \\
\hline organelle organization & $\mathrm{I}, 865(6.0 \%)$ & $2,574(5.0 \%)$ & $3.28 \mathrm{e}-44$ & $5.58 \mathrm{e}-42$ \\
\hline cell-cell signaling & $\mathrm{I}, \mathrm{II}(3.6 \%)$ & $\mathrm{I}, 475(2.9 \%)$ & $6.47 \mathrm{e}-40$ & $1.10 \mathrm{e}-37$ \\
\hline cellular developmental process & $1,993(6.5 \%)$ & $2,803(5.4 \%)$ & $1.02 \mathrm{e}-38$ & $1.73 \mathrm{e}-36$ \\
\hline cell communication & $8,167(26.5 \%)$ & $12,684(24.5 \%)$ & $7.68 \mathrm{e}-37$ & $1.30 \mathrm{e}-34$ \\
\hline signaling & 8,167 (26.5\%) & $12,684(24.5 \%)$ & $7.68 \mathrm{e}-37$ & $1.30 \mathrm{e}-34$ \\
\hline single organism signaling & $8,167(26.5 \%)$ & $12,684(24.5 \%)$ & $7.68 \mathrm{e}-37$ & $1.30 \mathrm{e}-34$ \\
\hline biological regulation & $8,400(27.2 \%)$ & $13,094(25.3 \%)$ & $8.48 \mathrm{e}-35$ & $1.44 \mathrm{e}-32$ \\
\hline
\end{tabular}

Note: Terms from the process ontology with $P$-value as good or better than $\mathrm{I}$.

Abbreviation: GO, Gene Ontology.

Table 6 KEGG pathway analysis for the predicted targets of the differentially expressed miRNAs

\begin{tabular}{|c|c|c|c|c|c|}
\hline Pathway & $\begin{array}{l}\text { Target genes } \\
\text { with pathway } \\
\text { annotation } \\
(15,068)\end{array}$ & $\begin{array}{l}\text { All genes of the } \\
\text { species with } \\
\text { pathway annotation } \\
(31,075)\end{array}$ & $P$-value & Q-value & $\begin{array}{l}\text { Pathway } \\
\text { ID }\end{array}$ \\
\hline Vascular smooth muscle contraction & $339(2.25 \%)$ & $551(1.77 \%)$ & $3.817347 \mathrm{e}-10$ & $6.353478 \mathrm{e}-08$ & ko04270 \\
\hline Pathways in cancer & $\mathrm{I}, 004(6.66 \%)$ & $1,810(5.82 \%)$ & $5.294565 \mathrm{e}-10$ & $6.353478 \mathrm{e}-08$ & ko05200 \\
\hline Glutamatergic synapse & $336(2.23 \%)$ & $559(1.8 \%)$ & $1.744324 \mathrm{e}-08$ & $1.122107 \mathrm{e}-06$ & ko04724 \\
\hline Folate biosynthesis & $44(0.29 \%)$ & $5 I(0.16 \%)$ & $1.870179 \mathrm{e}-08$ & $1.122107 \mathrm{e}-06$ & ko00790 \\
\hline Cytokine-cytokine receptor interaction & $524(3.48 \%)$ & $913(2.94 \%)$ & $2.741697 \mathrm{e}-08$ & $1.316015 \mathrm{e}-06$ & ko04060 \\
\hline Calcium signaling pathway & 477 (3.17\%) & $826(2.66 \%)$ & $4.015428 \mathrm{e}-08$ & $1.606171 \mathrm{e}-06$ & ko04020 \\
\hline Neuroactive ligand-receptor interaction & $544(3.61 \%)$ & 961 (3.09\%) & $1.834706 \mathrm{e}-07$ & $6.290421 \mathrm{e}-06$ & ko04080 \\
\hline Chemokine signaling pathway & 465 (3.09\%) & $813(2.62 \%)$ & $2.84434 \mathrm{Ie}-07$ & $8.533023 \mathrm{e}-06$ & ko04062 \\
\hline Aldosterone-regulated sodium reabsorption & $121(0.8 \%)$ & $18 \mid(0.58 \%)$ & $4.371058 \mathrm{e}-07$ & $1.165615 \mathrm{e}-05$ & ko04960 \\
\hline Salivary secretion & $233(1.55 \%)$ & $383(1.23 \%)$ & $7.00150 \mathrm{Ie}-07$ & $1.680360 \mathrm{e}-05$ & ko04970 \\
\hline Axon guidance & $385(2.56 \%)$ & $667(2.15 \%)$ & $8.425047 \mathrm{e}-07$ & $1.838192 \mathrm{e}-05$ & ko04360 \\
\hline Fc gamma R-mediated phagocytosis & $270(1.79 \%)$ & $455(\mathrm{I} .46 \%)$ & $1.864193 \mathrm{e}-06$ & $3.728386 \mathrm{e}-05$ & ko04666 \\
\hline GnRH signaling pathway & $274(1.82 \%)$ & $467(1.5 \%)$ & $5.521091 \mathrm{e}-06$ & $1.019278 \mathrm{e}-04$ & ko049I2 \\
\hline Melanogenesis & $260(1.73 \%)$ & $442(1.42 \%)$ & $7.238362 \mathrm{e}-06$ & $1.240862 \mathrm{e}-04$ & ko04916 \\
\hline Progesterone-mediated oocyte maturation & 290 (1.92\%) & $500(1.61 \%)$ & $1.073179 \mathrm{e}-05$ & $1.717086 \mathrm{e}-04$ & ko049I4 \\
\hline Prostate cancer & $338(2.24 \%)$ & $592(1.91 \%)$ & $1.387196 \mathrm{e}-05$ & $2.080794 \mathrm{e}-04$ & ko052I5 \\
\hline Regulation of actin cytoskeleton & $584(3.88 \%)$ & $1,066(3.43 \%)$ & $1.634052 \mathrm{e}-05$ & $2.306897 \mathrm{e}-04$ & ko048I0 \\
\hline Phosphatidylinositol signaling system & $236(1.57 \%)$ & $402(1.29 \%)$ & $2.244263 \mathrm{e}-05$ & $2.99235 \mathrm{Ie}-04$ & ko04070 \\
\hline ErbB signaling pathway & 294 (1.95\%) & $513(1.65 \%)$ & $3.313052 \mathrm{e}-05$ & $4.184908 \mathrm{e}-04$ & ko040I2 \\
\hline Oocyte meiosis & $328(2.18 \%)$ & $578(1.86 \%)$ & $3.589737 \mathrm{e}-05$ & $4.20409 \mathrm{Ie}-04$ & ko04II4 \\
\hline
\end{tabular}

Note: The significantly enriched pathways annotation for the predicted targets of the differentially expressed miRNAs following Smad4 reexpression.

Abbreviation: GnRH, gonadotropin-releasing hormone; KEGG, Kyoto Encyclopedia of Genes and Genomes. 
A SW480 SW620 RKO HCT116 LOVO HCT8

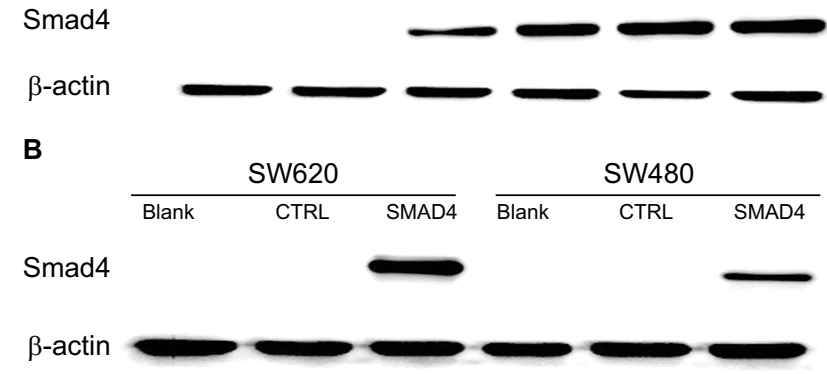

Figure 3 Generated stable Smad4 reexpression in human colon cancer cells. Notes: (A) Expression of Smad4 in six kinds of colon cancer cells including SW480, SW620, RKO, HCTII6, LOVO, and HCT8 by WB. (B) WB detection of Smad4 expression level in SW480 and SW620 cells following Smad4 reexpression. $\beta$-Actin was used as an internal control. Blank: null SW620 or SW480 cells. CTRL: empty vector-infected SW620 or SW480 cells. SMAD4: Smad4 vector-infected SW620 or SW480 cells.

Abbreviation: WB, Western blot.

\section{Discussion}

Colon cancer metastasis is the leading cause of death in colon cancer patients. ${ }^{13}$ However, the molecular mechanism is not clear. Previous studies have shown that SMAD4 is strongly associated with the development of colon metastases, which plays the antimetastatic role in colon cancer metastasis. ${ }^{14-16}$ Smad4, known as deleted in pancreatic carcinoma, locus 4 (DPC4), was first identified on the basis of frequent homozygous deletions and mutations affecting 18q21.1 in the pancreatic tumor, and was found to be involved in the TGF- $\beta 1$ signaling pathway. ${ }^{17}$ The Smad4 was deregulated in many cancers and was demonstrated to mediate tumor process including pancreatic cancer, hepatocellular carcinoma, and so on. ${ }^{17}$ Some researchers have shown that Smad 4 could inhibit the activity of wingless/integrated (WNT) $/ \beta$-catenin signaling pathway in cancer, ${ }^{10}$ inhibit the EMT process ${ }^{9,10}$ and changes the immune microenvironment, ${ }^{1,14,16}$ which are involved in the tumor development and process.

In this study, we also demonstrate that Smad4 can regulate the migration and invasion abilities of colon cancer. However, the exact mechanism of Smad4-mediated tumor metastasis remains to be further investigated. Many studies indicate that miRNAs have important functions in tumor progression, especially recurrence, drug resistance, and metastasis. ${ }^{4,5,8}$ Ahn et al reported that Smad3 regulated, at the transcriptional level, miR-200 family members, which themselves regulate ZEB1 and ZEB2, known as transcriptional repressors of E-cad, at the posttranscriptional level in a TGF- $\beta$-independent manner, which regulate EMT in gastric cancer cells. ${ }^{18}$ Davis et al think that Smad proteins bind a conserved RNA sequence to promote miRNA maturation by Drosha. They think that Smads are multifunctional proteins that modulate gene expression transcriptionally through DNA binding and post-transcriptionally through pri-miRNA binding and regulation of miRNA processing. ${ }^{19}$ Blahna and Hata reported that Smads modulated miRNA expression through both transcriptional and post-transcriptional mechanisms: 1) transcriptional regulation of miRNAs by Smads largely resembles the canonical TGF- $\beta$ signaling pathway; 2 ) posttranscriptional regulation of miRNA biogenesis acts on pri-miRNA in the nucleus..$^{20,21}$ Therefore, we hypothesize that Smad4 could regulate the expression of miRNA and Smad4-mediated activation or inhibition of miRNAs might influence colon cancer metastasis.

As far as we know, there are no reports about the miRNA expression profile following smad4 reexpression in a colon cancer cell model. In our previous study, we identify differentially expressed miRNAs in two colon carcinoma cell lines: SW480, derived from primary colon carcinoma, and SW620, derived from lymph node metastasis, which were obtained from the same patient. And it is the first systematic analysis of the differences of miRNAs between SW480 and SW620 cells, which demonstrated the potential miRNAs biomarkers for predicting colon cancer metastasis. ${ }^{22}$ In this study, we further study the role of miRNAs in colon cancer metastasis. First, we established the colon cancer cell lines with stable overexpression of Smad4. Then, we used the next-generation sequencing technology to identify the differentially expressed miRNAs in the SW620 colon cancer cell line transfected with smad4 lentiviral vector. We found Smad4 reexpression significantly upregulated 43 known miRNAs and downregulated 10 known miRNAs expression. Furthermore, pathway analysis of predicted miRNA targets showed that these genes were mainly involved in protein-binding transcription factor activity, vascular smooth muscle contraction, pathways in cancer metastasis, and PI3K-Akt signal pathway. We also performed qRT-PCR and WB to validate the partial results of sequencing. We demonstrated that restoration of Smad4 resulted in a marked decrease of Vim by inhibiting p-AKT and p-EPHA2, but significantly increased the E-cad by AKT-EPHA2 pathways in colon cancer SW480 cells. Many studies indicate that targeting key signaling components of PI3K-AKT pathway by restoring or inhibiting miRNA function holds promise as a potential therapeutic approach to suppress EMT in 
A

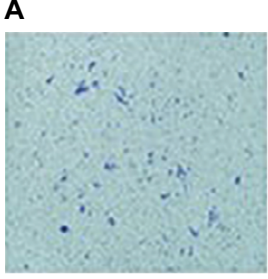

vec

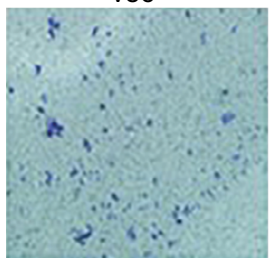

TGF- $\beta 1+$ Vec

B

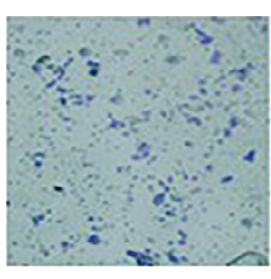

vec

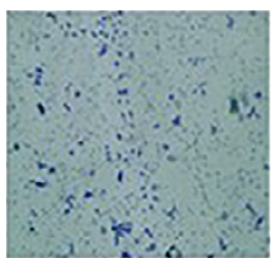

TGF $-\beta+$ Vec

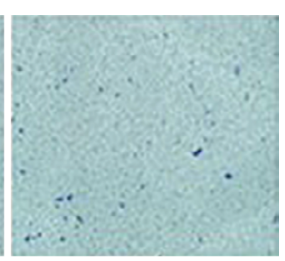

smad4

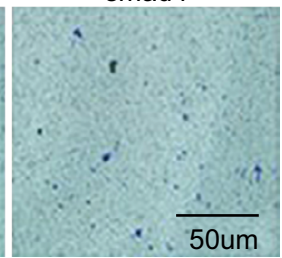

TGF- $\beta 1+$ smad4

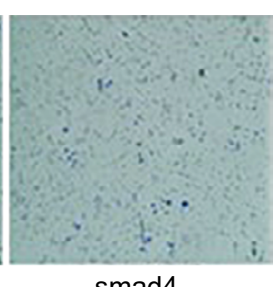

smad4

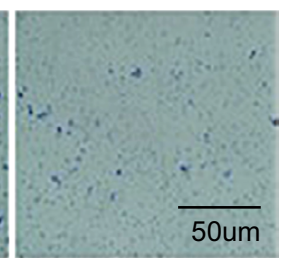

TGF- $\beta 1+$ smad4

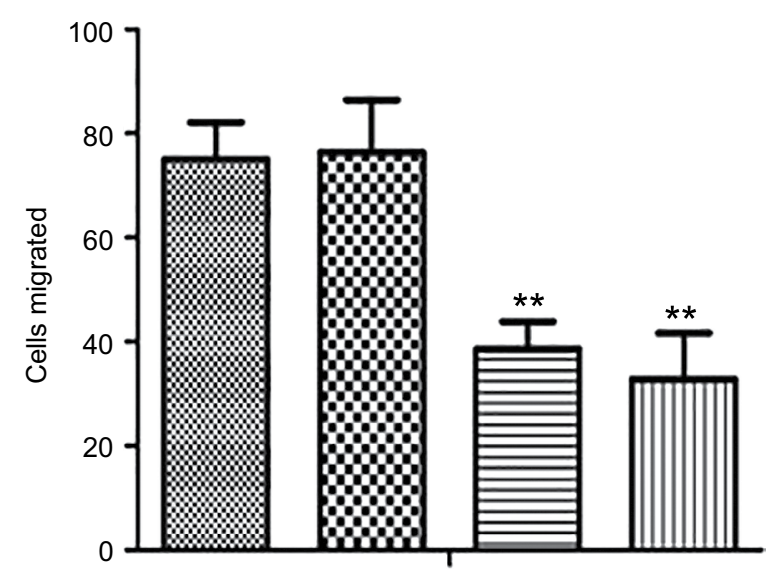

\%8 vec

Th $\mathrm{Tg}-\beta 1+\mathrm{Vec}$

smad4

III TGF- $\beta 1+$ smad 4

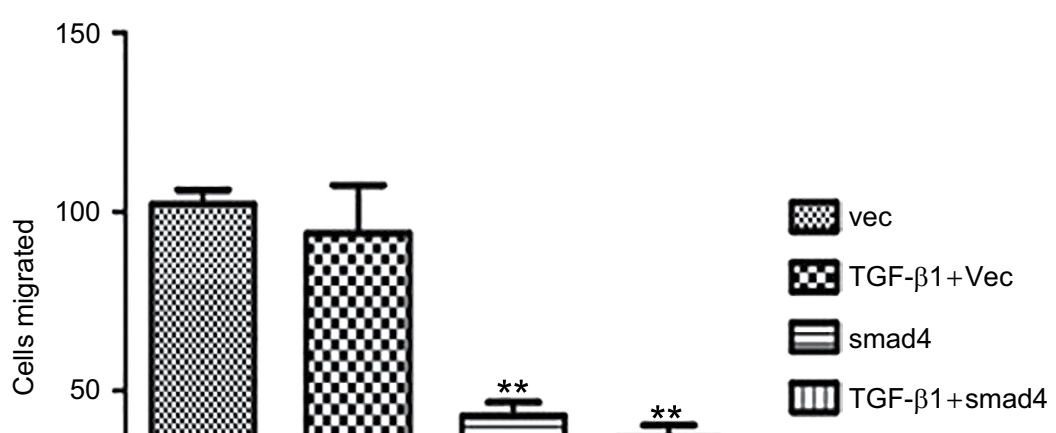

c

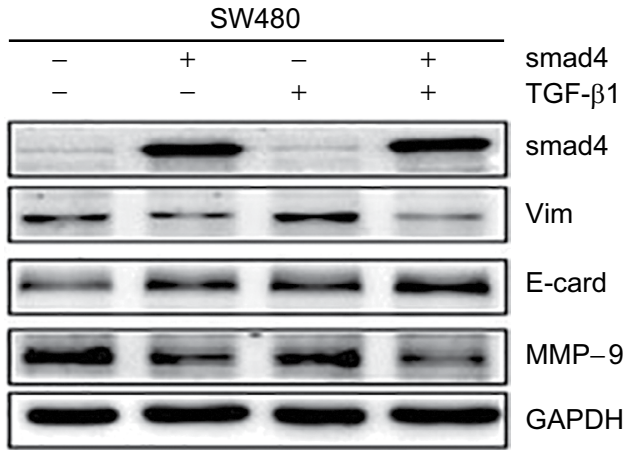

Figure 4 Migration and invasion assay in SW480 cells following stable Smad4 reexpression.

Notes: (A) Transwell cell migration and (B) invasion assay in Smad4-negative and Smad4-positive SW480 cells in the case of adding and without adding TGF- $\beta$ I. (C) Protein expression level of Smad4, E-cad, Vim, and MMP-9 by WB with or without TGF- $\beta$ I cytokine. GAPDH was used as an internal control. **P<0.0I.

Abbreviations: E-cad, E-cadherin; GAPDH, glyceraldehyde-3-phosphate dehydrogenase; MMP-9, Matrix Metalloproteinase-9; Vim, Vimentin; WB, Western blot.

cancer $^{23}$ and miRNAs could promote cell migration via the AKT signaling pathway. ${ }^{24}$

Therefore, we speculate that $\mathrm{Smad} 4$ could regulate the colon cancer metastasis via a new mechanism, that is, through
Smad4-miRNAs-downstream genes (such as AKT, EPHA2, E-cad, and Vim) axis. However, we should do further study to demonstrate how Smad4 regulated the expression of miRNA. We guess that Smad4 could directly mediate the miRNA 


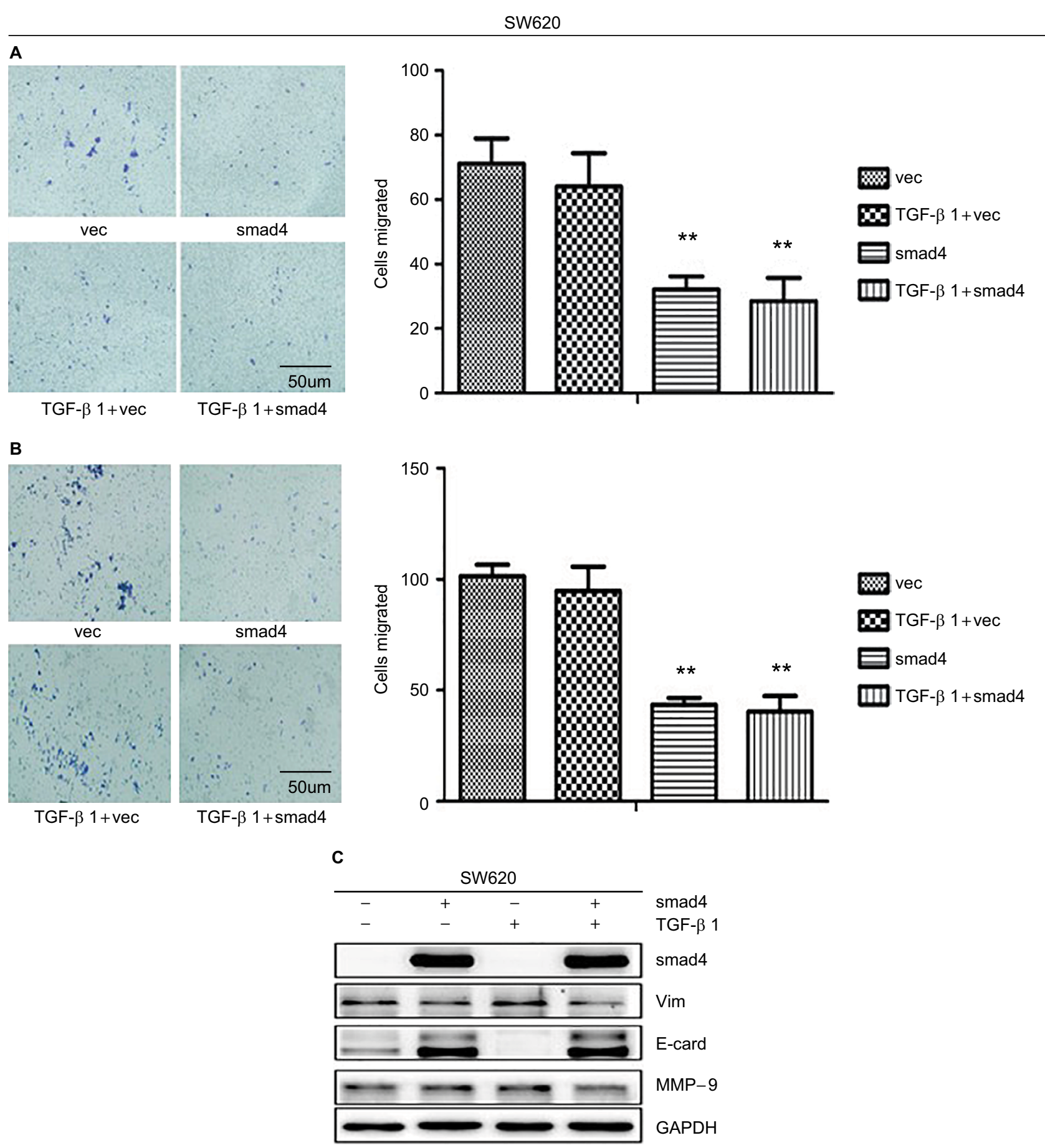

Figure 5 Migration and invasion assay in SW620 cells following stable Smad4 reexpression.

Notes: (A) Transwell cell migration and (B) invasion assay in Smad4 negative and positive SW620 cells with or without adding TGF- $\beta$ I. (C) WB detection of protein expression level of Smad4, E-cad, Vim, MMP-9 with or without TGF- $\beta$ I cytokine. GAPDH was used as an internal control. **P<0.01.

Abbreviations: E-cad, E-cadherin; MMP-9, Matrix Metalloproteinase-9; Vim, Vimentin; WB, Western blot.

expression through Smad-binding element by binding to the gene promoter zone or indirectly regulate the miRNAs expression. Of course it remains a deeply further study.

\section{Conclusion}

This is the first systematic report of Smad4-mediated miRNA expression profiling in Smad4-positive and Smad4-negative
SW620 human colon cancer cells, which may help us better understand the role of Smad4-mediating metastasis of colon cancer.

\section{Acknowledgments}

This work was supported by National Nature Science Foundation of China (Grant No. 81172285) and Natural Science 
A

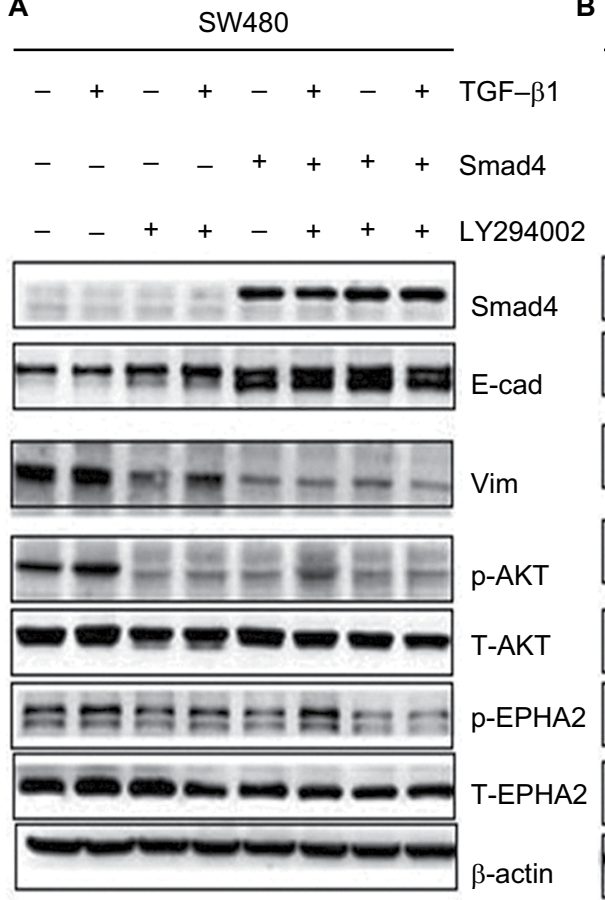

B

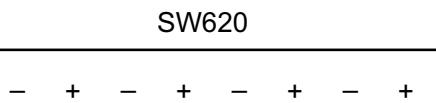

$-\quad-\quad++++$
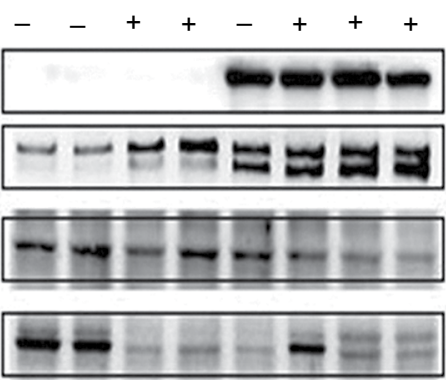

$-\infty=0$

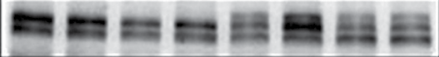

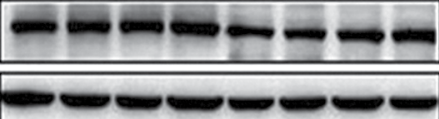

Figure 6 SMAD4 reexpression modulates E-cad and Vim expression by AKT-EPHA2 signal pathways.

Notes: (A) Restoration of SMAD4 results in a marked decrease of Vim by inhibiting P-AKT and P-EPHA2, but significantly increased the E-cad by AKT-EPHA2 pathways in colon cancer SW480 cells. (B) Restoration of SMAD4 results in the same phenomenon in colon cancer SW620 cells. Total protein extracted from indicated cells were lysed for WB analysis. WBs were then performed with the indicated antibodies. $\beta$-Actin served as an internal control.

Abbreviations: E-cad, E-cadherin; Vim, Vimentin; WB, Western blot.

Foundation of Fujian Province, People's Republic of China (Grant No. 2018J01392 and 2018J01390).

\section{Disclosure}

The authors report no conflicts of interest in this work.

\section{References}

1. Wasserman I, Lee LH, Shia J, et al. SMAD4 loss in colorectal cancer: correlation with recurrence, chemoresistance, and immune infiltrate. $J$ Clin Oncol. 2017;35(4_suppl):587-587.

2. Salovaara R, Roth S, Loukola A, et al. Frequent loss of SMAD4/DPC4 protein in colorectal cancers. Gut. 2002;51(1):56-59.

3. Ahmed M, Siraj AK, Masoodi T, Bu R, Al-Dayel F, Al-Kuraya KS. Abstract 2255: SMAD4 loss is associated with poor prognosis in Middle Eastern colorectal cancer. Cancer Res. 2017;77(13 Supplement):2255-2255.

4. Lopez-Camarillo C, Marchat LA, Arechaga-Ocampo E, et al. MetastamiRs: non-coding MicroRNAs driving cancer invasion and metastasis. Int J Mol Sci. 2012;13(2):1347-1379.

5. Pencheva N, Tavazoie SF. Control of metastatic progression by microRNA regulatory networks. Nat Cell Biol. 2013;15(6):546-554.

6. Davoodian P, Ravanshad M, Hosseini SY, et al. Effect of TGF- $\beta /$ smad signaling pathway blocking on expression profiles of miR-335, miR150, miR-194, miR-27a, and miR-199a of hepatic stellate cells (HSCs). Gastroenterol Hepatol Bed Bench. 2017;10(2):112-117.

7. Wu CW, Storey KB. Regulation of Smad mediated microRNA transcriptional response in ground squirrels during hibernation. Mol Cell Biochem. 2018;439(1-2):151-161.
8. Zhou J, Hu M, Wang F, Song M, Huang Q, Ge B. miR-224 Controls human colorectal cancer cell line HCT116 proliferation by targeting Smad4. Int J Med Sci. 2017;14(10):937-942.

9. Zhang B, Halder SK, Kashikar ND, et al. Antimetastatic role of Smad4 signaling in colorectal cancer. Gastroenterology. 2010;138(3):969-980.

10. Shiou SR, Singh AB, Moorthy K, et al. Smad4 regulates claudin-1 expression in a transforming growth factor-beta-independent manner in colon cancer cells. Cancer Res. 2007;67(4):1571-1579.

11. Papageorgis P, Cheng K, Ozturk S, et al. Smad4 inactivation promotes malignancy and drug resistance of colon cancer. Cancer Res. 2011;71(3):998-1008.

12. Yamamoto T, Kawada K, Itatani Y, et al. Loss of SMAD4 promotes lung metastasis of colorectal cancer by accumulation of CCR 1+ tumorassociated neutrophils through CCL15-CCR1 axis. Clin Cancer Res. 2017;23(3):833-844.

13. Voorneveld PW, Kodach LL, Jacobs RJ, et al. Loss of SMAD4 alters BMP signaling to promote colorectal cancer cell metastasis via activation of Rho and ROCK. Gastroenterology. 2014;147(1):196-208.

14. Itatani Y, Kawada K, Fujishita T, et al. Loss of SMAD4 from colorectal cancer cells promotes CCL15 expression to recruit CCR1+ myeloid cells and facilitate liver metastasis. Gastroenterology. 2013;145(5):1064-1075.

15. Isaksson-Mettävainio M, Palmqvist R, Forssell J, Stenling R, Oberg A. SMAD4/DPC4 expression and prognosis in human colorectal cancer. Anticancer Res. 2006;26(1B):507-510.

16. Kitamura T, Kometani K, Hashida H, et al. SMAD4-deficient intestinal tumors recruit CCR1+ myeloid cells that promote invasion. Nat Genet. 2007;39(4):467-475.

17. Chen YW, Hsiao PJ, Weng CC, et al. SMAD4 loss triggers the phenotypic changes of pancreatic ductal adenocarcinoma cells. BMC Cancer. 2014; 14:181. 
18. Ahn SM, Cha JY, Kim J, et al. Smad3 regulates E-cadherin via miRNA200 pathway. Oncogene. 2012;31(25):3051-3059.

19. Davis BN, Hilyard AC, Nguyen PH, Lagna G, Hata A. Smad proteins bind a conserved RNA sequence to promote microRNA maturation by Drosha. Mol Cell. 2010;39(3):373-384.

20. Blahna MT, Hata A. Smad-mediated regulation of microRNA biosynthesis. FEBS Lett. 2012;586(14):1906-1912.

21. Davis-Dusenbery BN, Hata A. Smad-mediated miRNA processing: a critical role for a conserved RNA sequence. RNA Biol. 2011;8(1):71-76.

22. Yan W, Yang W, Liu Z, Wu G. Characterization of microRNA expression in primary human colon adenocarcinoma cells (SW480) and their lymph node metastatic derivatives (SW620). Onco Targets Ther. 2018;11:4701-4709.
23. Dong P, Konno Y, Watari H, Hosaka M, Noguchi M, Sakuragi N. The impact of microRNA-mediated PI3K/AKT signaling on epithelialmesenchymal transition and cancer stemness in endometrial cancer. J Transl Med. 2014;12:231.

24. Guo J, Xu L, Teng X, Sun M. MicroRNA-7-5p regulates the proliferation and migration of intestinal epithelial cells by targeting trefoil factor 3 via inhibiting the phosphoinositide 3-kinase/Akt signalling pathway. Int J Mol Med. 2017;40(5):1435-1443.

25. Zhongcai L, Wei Y, Fusheng L. Over-expression of Smad4 could prevent migration and invasion in colon cancer SW480 and SW620 cells and mechanism. Chin J Exp Surg. 2018;35(2):238-241.
Cancer Management and Research

\section{Publish your work in this journal}

Cancer Management and Research is an international, peer-reviewed open access journal focusing on cancer research and the optimal use of preventative and integrated treatment interventions to achieve improved outcomes, enhanced survival and quality of life for the cancer patient. The manuscript management system is completely online and includes
Dovepress

a very quick and fair peer-review system, which is all easy to use. Visit http://www.dovepress.com/testimonials.php to read real quotes from published authors. 